\title{
Evaluación y selección de líneas avanzadas de arroz (Oriza sativa L.) con alto contenido de zinc en condiciones de riego y secano en Nicaragua
}

\section{Evaluation and selection of advanced lines rice (Oryza sativa L.) with high Zinc content in Irriga- tion and rainfed conditions in Nicaragua}

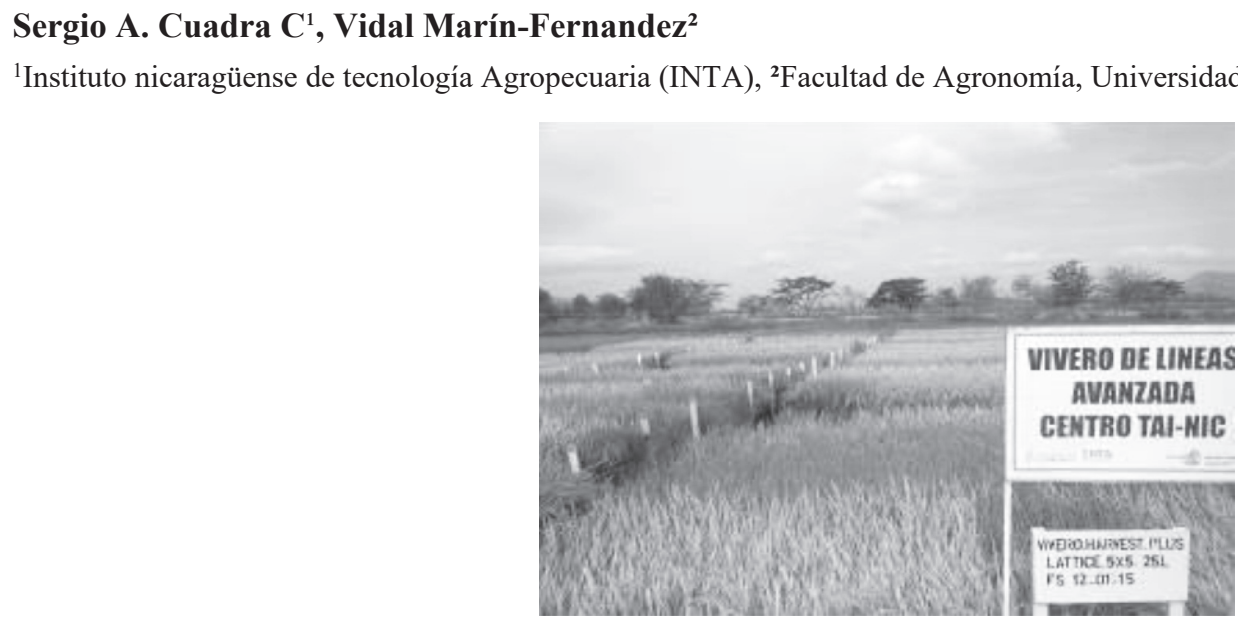

\section{RESUMEN}

La bioacumulación de $\mathrm{Zn}$ en el endosperma de cultivos de alto consumo popular como el arroz tiene gran importancia para resolver problemas de carácter nutricional. La presente investigación consisió en distintas fases de evaluación-selección de líneas avanzadas de arroz con alto contenido de zinc (a nivel de vivero, evaluación de líneas avanzadas y evaluación multi-ambiental en diferentes sitios del país). Los experimentos se establecieron en condiciones de riego y secano en los años 2014 y 2015, en las siguintes localidades: Centro Experimental de Arroz "TAI-NIC", Jalapa, Posoltega, Masatepe, San Carlos, Pantasma y Dario. El Diseño empleado fue en Bloques Incompletos No Balanceados (Alpha Latice). Se midieron datos de comportamiento agronómico (días a madures, altura de planta, macollamiento, longitud de panícula, peso de grano, rendimiento), calidad de grano y contenido de zinc en el grano pulido mediante la metodología de fluorescencia de rayos X (FRX). De los genotipos evaluados se seleccionaron cuatro que combinan características agronómicas, industriales y nutricionales deseables. Se estableció una relación positiva entre la cantidad de zinc disponible en el suelo y la cantidad de zinc encontrada en grano pulido. Se determinaron diferencias significativas entre los genotipos, ambientes e interacción genotipo*ambiente. Se seleccionaron genotipos que presentan un comportamiento adaptativo amplio y genotipos con adaptación específica para cada condición agroclimática y de manejo. Se identificaron dos mega-ambientes con genotipos en común. En el primero formado por los sitios de Pantasma y Jalapa destaca el genotipo IR31917-45-3-2-1-1SR-5-M (T4). En el segundo mega-ambiente formado por los sitios de Masatepe y San Carlos destaca el genotipo CT18504-4-5-3Vi-2-3P (T14). En los ambientes únicos de Posoltega y Darío los mejores genotipos fueron IR758705-8-5-B-2-B-1SR-8-M (T6) para el primero y CT19298-(100)-1-23-1-4MP (T1) para el segundo. La variable que más influenció la producción fue días a madures, confirmando la tendencia de que a ciclo más largo, existe mayor rendimiento; y longitud de panícula.

Palabras clave: nutrición, genotipo, ambiente, grano, suelo, fluorescencia de rayos $\mathrm{X}$.

\begin{abstract}
The bioaccumulation of $\mathrm{Zn}$ in the endosperm of popular high consumption crops such as rice has great importance to solve nutritional problems. The present research consisted of different stages of evaluation-selection of advanced rice lines with high zinc content (nursery level, evaluation of advanced lines and multi-environmental evaluation). The experiments were established under irrigation and rainfed conditions in years 2014 and 2015, in the following locations: rice experimental center "TAI-NIC", Jalapa, Posoltega, Masatepe, San Carlos, Pantasma and Dario. The experimental design was Unbalanced Uncomplete Blocks (Alpha Latice). Agronomic performance data (days to maturity, plant height, tillering, panicle length, grain weight, yield), grain quality and zinc content in the polished grain were measured by X-ray fluorescence (FRX) methodology. Four genotypes that combine desirable agronomic, industrial and nutritional characteristics were selected. A positive relationship was established between the amount of zinc available in the soil and the amount of zinc found in polished grain. Significant differences were determined between genotypes, environments and genotype * environment interaction. Genotypes that exhibit a broad adaptive behavior and genotypes with specific adaptation for each agro climatic and management condition were selected. Two mega-environments with genotypes in common were identified. The first genotype, IR31917-45-3-2-1-1SR-5-M (T4), was formed by Pantasma and Jalapa sites. The second mega-environment was formed by Masatepe and San Carlos sites, with the genotype CT18504-4-5-3Vi-2-3P (T14). In the unique environments of Posoltega and Darío the best genotypes were IR75870-5-8-5-B-2-B1SR-8-M (T6) for the first and CT19298- (100) -1-2-3- 1-4MP (T1) for the second. The variable that most influenced the production was days to maturity, confirming the tendency that at a longer cycle, there is higher yield; and panicle length.
\end{abstract}

Keywords: nutrition, zinc, grain, soil, X-ray fluorescence autorizaciones adicionales a las aquí delimitadas se pueden obtener en el correo freddy.aleman@ci.una.edu.ni C Copyright 2017. Universidad Nacional Agraria 


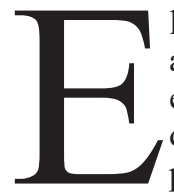
1 arroz (Oriza sativa L.) es la principal fuente de alimento para miles de millones de personas en el mundo. En Nicaragua el consumo per cápita de arroz casi se duplicó en los últimos diez años, pasando de $29.5 \mathrm{~kg}$ a $50 \mathrm{~kg}$; parte importante de este consumo se satisface con producción nacional. Durante el ciclo agrícola 2012/13, se cultivaron aproximadamente 101148 hectáreas de arroz, con una producción de 268659 toneladas de arroz paddy o granza y un rendimiento promedio de $2.65 \mathrm{t} \mathrm{ha-}^{-1}$ (MAG, 2013), para una producción de 240 mil toneladas anualmente de arroz oro. El déficit nacional de arroz es aproximadamente el $20 \%$.

En las poblaciones de los países en desarrollo, existen altas deficiencias en zinc, hierro y vitamina A; la deficiencia de zinc es reportada como la quinta causa principal de las enfermedades y muertes en estos países. Los problemas de salud causados por la deficiencia de zinc incluyen anorexia, enanismo, sistema inmunológico débil, lesiones en la piel, hipogonadismo y diarrea (McClain, 1985). Se estima que hombres entre 15 y 74 años necesitan alrededor de 12 a $15 \mathrm{mg}$ de zinc al día, mientras que las mujeres de la misma edad, requieren alrededor de $68 \mathrm{mg}$ de zinc por día (Sandstead, 1985).

En regiones donde la población hace uso masivo de arroz como base de la alimentación, presentan problemas nutricionales relacionados con deficiencias en minerales ( $\mathrm{Fe}$ y $\mathrm{Zn}$ ), vitaminas, anemia y ceguera. Los niños, ancianos y mujeres embarazadas son los más afectados y vulnerables. Según Pachón (2007), en meso América el 28\% de las mujeres son anémicas y del 20 al 40\% de la niñez padecen del mismo déficit.

Las estrategias empleadas para combatir la malnutrición no han dado los resultados esperados. Datos de reciente publicación indican que el fitomejoramiento constituye una herramienta eficiente, confiable y de menor costo para el desarrollo de germoplasmas con mayor valor nutricional (Martínez, 2007). Lo anterior justifica el incremento del interés que ha adquirido el estudio de la bioacumulación de $\mathrm{Zn}$ en el endosperma de cultivo de alto consumo popular como el arroz.

En años recientes se han liberado en Bolivia, Cuba y Panamá variedades con alto contenido nutricional (Azucena, Saavedra 27, IACUBA 30, IDIAP GAB 2, IDIAP GAB 6, IDIAP GAB 8 y IDIAP GAB 11). El contenido de zinc en el endosperma de estas variedades oscila entre 13.2 y $18.6 \mathrm{mg}$ $\mathrm{k}-^{1}$.

Este trabajo es parte de las estrategias desarrolladas para contribuir al mejoramiento de la seguridad alimentaria, la nutrición y la salud en las regiones de Nicaragua con mayor prevalencia de desnutrición. Se tiene como objetivo el mejorar el valor nutricional del arroz, aumentando su contenido de zinc en el grano pulido (de una línea base de 16 ppm hasta $28 \mathrm{ppm}$ ), manteniendo sus buenas calidades agronómicas como rendimiento, resistencia a enfermedades, calidad de grano y molinera.

\section{MATERIALES Y MÉTODOS}

Ochenta y seis líneas mejoradas con alto contenido de zinc, provenientes de cruces convencionales, cruces interespecíficos y de mejoramiento poblacional, tanto de condiciones de riego como de secano favorecido, fueron introducidas a Nicaragua desde el CIAT-Colombia para ser evaluadas y seleccionadas durante tres ciclos de siembra consecutivos entre el período de 2014 al 2015. Los municipios donde se establecieron los experimentos fueron Dario, Pantasma, Jalapa, Posoltega, Masatepe y San Carlos.

Estos ensayos se establecieron en un diseño de Bloques Incompletos No Balanceado (Alpha Latice), con distancias de siembra de $0.25 \mathrm{~m}$ entre surco, la parcela experimental consistió de 4 surcos de 5 metros de largo. Se midieron datos de comportamiento agronómico (rendimiento, resistencia a enfermedades, calidad de grano y molinera) y contenido de zinc en el grano pulido mediante la metodología de fluorescencia de rayos $\mathrm{X}$ (FRX). En cada ensayo se registraron datos climáticos (precipitación y temperatura), monitoreo y manejo de plagas y enfermedades, y análisis químico de los suelos.

En Darío, Jalapa, Posoltega, Masatepe y Pantasma, la preparación del suelo fue mediante tracción mecánica (arado y gradeo) y la siembra en surco a chorrillo (semilla, 130 $\mathrm{kg}$ ha-1). Solamente en San Carlos, se preparó con tracción animal y la siembra fue al espeque (semilla, $51 \mathrm{~kg}$ ha-1).

En todos los ensayos el día de la siembra, se aplicó al fondo del surco fertilizante de la fórmula18-46-00a razón de $130 \mathrm{~kg}$ ha-1más65 kg ha-1 de MOP (00-00-60). A los $15 \mathrm{y}$ 35 días después de la emergencia (dde), se aplicó Urea 46\% a razón de $130 \mathrm{~kg}$ ha-1 por fraccionamiento. A los 55 dde, se realizó un último fraccionamiento de UREA 46\%, en dosis de $65 \mathrm{~kg}$ ha-1 más $65 \mathrm{~kg}$ ha-1 de MOP (00-00-60). En total se aplicó 173-60-39 kg ha-1 de N-P2O5-K2O respectivamente.

Los resultados experimentales se obtuvieron en condiciones de suelos pardos oscuros, arcillosos y profundos $(60 \mathrm{~cm})$, con buen drenaje y con $\mathrm{pH}$ ligeramente alcalino. Estos suelos presentan contenidos medios en nitrógeno, altos en fósforo, potasio, calcio y magnesio y bajos en zinc.

Se realizó el Análisis de Varianza para probar la hipótesis de igualdad entre medias de tratamientos. Se utilizó la prueba de separación de medias de rangos múltiples de Tukey al 5\% de significancía $(\mathrm{P}=0.05)$. También se realizaron Análisis de Regresión por Genotipo, Multiambiental, Frecuencia, Regresión, Correlación y Conglomerado. Los programas usados fueron INFOSTAT e INFO GEN. La normalidad y homogeneidad de datos fue comprobada mediante las pruebas de Shapiro-Wlilk y Levene. Se aplicaron transformaciones en datos que no correspondían con los supuestos del ANDEVA.

\section{RESULTADOS Y DISCUSIÓN}

Primera fase: Evaluación y selección en etapa de vivero. El análisis de calidad nutricional determinó que el 38\% de los 91 genotipos superan los $16 \mathrm{mg} \mathrm{kg-}^{1}$ de $\mathrm{Zn}$ en el grano pulido. La media general fue de $15.3 \mathrm{mg} / \mathrm{kg}$ zinc, con valores mínimos y máximos de 11.9 y $20.3 \mathrm{mg} \mathrm{kg-}^{1}$. Cuatro de los 14 genotipos que superan productivamente al testigo (INTA L-9), presentan datos mayores y estables en contenidos de Zinc. Estos materiales no difieren estadísticamente con las 
líneas de mayor contenido nutricional evaluadas en el vivero. En el vivero se identificaron un total de 10 líneas con contenidos de zinc que fluctúan entre 18 y $20.3 \mathrm{mg} \mathrm{kg-}^{1}$, sin embargo, su comportamiento productivo es bajo. En Nicaragua, el promedio en el contenido de $\mathrm{Zn}$ de las variedades comerciales de arroz es de $16 \mathrm{mg} \mathrm{kg-}^{1}$, lo cual constituye la línea base a superar.

Estos materiales podrían ser la base de futuros cruzamientos que permitan desarrollar un programa de mejoramiento convencional incluyendo métodos de selección masal, pedigree, retrocuzamiento, mejoramiento poblacional y mutagénesis para obtener alto contenido de zinc en el grano pulido. La figura 1 indica que el 38\% de los genotipos superan los $16 \mathrm{mg} \mathrm{kg-}^{1}$ de zinc en el grano pulido. La media general fue de $15.3 \mathrm{mg} \mathrm{kg-}^{1}$ de zinc con valores mínimos y máximos de 11.9 y $20.3 \mathrm{mg} \mathrm{kg-}{ }^{1}$.

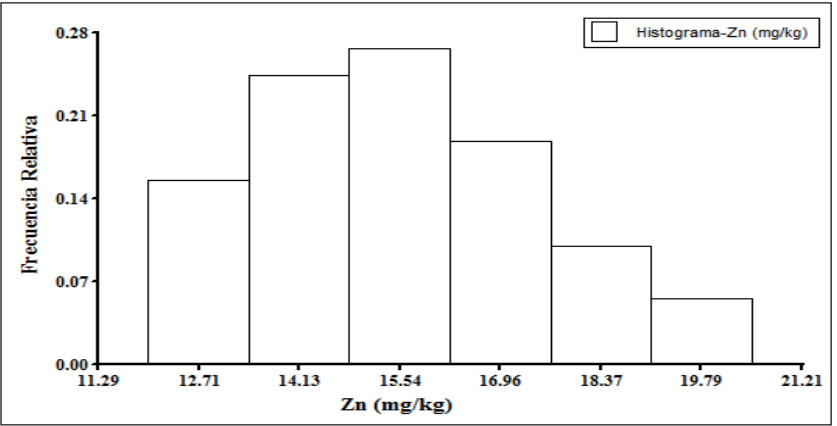

Figura 1. Frecuencia relativa del contenido de $\mathrm{Zn}\left(\mathrm{mgkg}^{1}\right)$ en el grano pulido de líneas evaluadas en el vivero TAINIC, períodolluvioso 2014

Datos obtenidos por Choudhury (1991), indican que las condiciones climáticas y de suelos influyen en la expresión del contenido de zinc en el grano de arroz. Estudios realizados por Borrero et al., (2014) y por Nova et al., (2008), relacionan que la expresión del zinc en el grano pulido además del carácter genético depende de factores como el manejo, suelo y clima.

En el suelo el contenido de zinc soluble en agua, disminuye con la elevación de $\mathrm{pH}$. Un alto $\mathrm{pH}$ del suelo está correlacionado con la disminución del zinc en el tejido vegetal. El rango más común de $\mathrm{Zn}$ total en el suelo se sitúa entre 10 y $300 \mathrm{mg} \mathrm{kg}-1$ con una media de $50 \mathrm{mg}$ kg-1 (Goldschmidt, 1954).

Sobre la base de lo anterior se debe considerar que, los contenidos disponibles de zinc en el suelo del TAINIC son bajos y el pH ligeramente alcalino, siendo estas condiciones determinantes para que los contenidos de zinc en el grano fueran también entre bajos y medios.

En este experimento se determinó que el contenido de zinc en el grano está fuertemente vinculado al contenido del elemento en suelo (figura 2). El análisis de regresión con un coeficiente de determinación $\mathrm{R} 2=74 \%$, explica que la variabilidad en el contenido de zinc en el grano está influenciada por el contenido de zinc en el suelo, mostrando una tendencia que no logra determinar el punto de inflexión, de que a mayor contenido de zinc en el suelo, se incrementa el contenido del mismo en el grano pulido. La disponibilidad de zinc en el suelo durante el estudio son consideradas bajas, infiriendo que al menos el 38\% de las líneas presentan alta capacidad de absorción por su naturaleza genética.

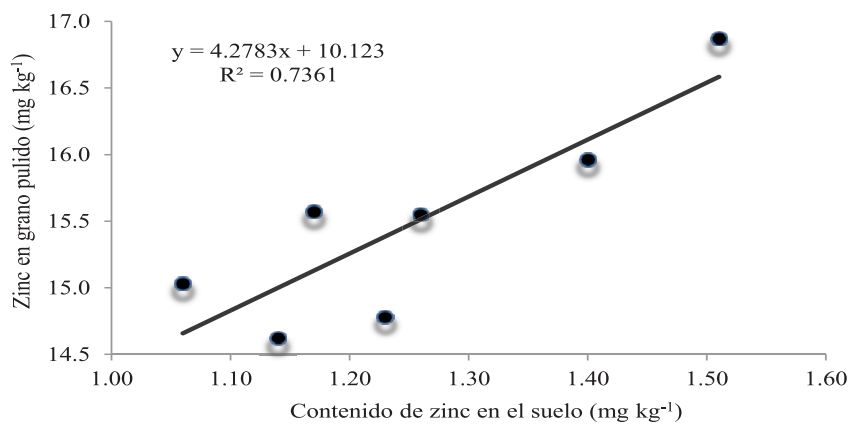

Figura 2. Correlación entre el contenido de zinc en el suelo y el contenido en el grano pulido de arroz, vivero TAINIC período lluvioso 2014.

Se identificaron 14 materiales promisorios que superan productivamente al testigo INTA L-9 (cuadro 1). El $15 \%$ de los materiales introducidos superaron productivamente al testigo INTA L-9 y solamente el uno por ciento superó al testigo universal (IR 64). Los materiales promisorios que superaron productivamente y cualitativamente a INTA L-9 son los seleccionados para conformar la siguiente fase de investigación: Evaluación de líneas avanzadas de arroz.

Cuadro 1. Comportamiento agronómico, nutricional e industrial de los mejores materiales productivos. Vivero en el TAINIC, período lluvioso 2014

\begin{tabular}{|c|c|c|c|c|c|c|c|c|}
\hline \multirow{2}{*}{ Trat } & \multirow{2}{*}{ Genotipo } & \multirow{2}{*}{\multicolumn{2}{|c|}{$\frac{\text { Rend. } 14 \%{ }^{\circ} \mathrm{H}}{\mathrm{kg} \mathrm{ha}^{1}}$}} & \multicolumn{3}{|c|}{ Contenido de $\mathrm{Zn}$} & \multicolumn{2}{|c|}{ Calidad industrial } \\
\hline & & & & \multicolumn{2}{|c|}{$\mathrm{mg} \mathrm{kg}^{1}$} & \multirow{2}{*}{$\begin{array}{r}\% \mathrm{CV} \\
4.8\end{array}$} & \multirow{2}{*}{$\begin{array}{r}\% \text { Oro } \\
66\end{array}$} & \multirow{2}{*}{$\frac{\mathrm{R} E / \mathrm{Q}}{90 / 10}$} \\
\hline 37 & IR90154-53-2-1-M & 10931 & $\mathrm{~A}^{*}$ & 13.5 & F-K & & & \\
\hline 98 & IR 64 & 10166 & $\mathrm{AB}$ & 13.6 & F-K & 8.8 & 68 & $93 / 07$ \\
\hline 29 & IR75870-5-8-5-B-2-B-1SR-8-M & 9464 & $\mathrm{AB}$ & 13.2 & G-K & 3.6 & 68 & $91 / 09$ \\
\hline 39 & IR90140-47-3-M-2SR-1-M & 9320 & $\mathrm{AB}$ & 16.3 & A-K & 17.8 & 68 & $96 / 04$ \\
\hline 2 & 926 & 9235 & $\mathrm{AB}$ & 14.1 & E-K & 6.7 & 66 & $90 / 10$ \\
\hline 34 & IR77378-11-B-6-1-B-1SR-3-M & 9141 & $\mathrm{AB}$ & 12.3 & $\mathrm{~K}$ & 10.8 & 65 & $85 / 15$ \\
\hline 67 & PCT-11 $\backslash 0 \backslash 02$, Bo $\backslash 1 \backslash 1>58-1-1-3-1-\mathrm{M}$ & 9096 & $\mathrm{AB}$ & 13.8 & F-K & 4.0 & 69 & $95 / 05$ \\
\hline 36 & IR90154-53-2-2-M & 9064 & $\mathrm{AB}$ & 13.4 & F-K & 3.1 & 67 & $94 / 06$ \\
\hline 28 & IR87233-19-4-2-2-M & 8988 & $\mathrm{AB}$ & 13.5 & F-K & 3.5 & 69 & $96 / 04$ \\
\hline 20 & IR87233-135-2-3-3-M & 8936 & $\mathrm{AB}$ & 13.3 & F-K & 8.6 & 67 & $95 / 05$ \\
\hline 79 & РCT-11 $10 \backslash 02$, Bo $\backslash 2 \backslash 1>46-M-3-2-4-2-M$ & 8875 & $\mathrm{AB}$ & 14.8 & B-K & 11.2 & 67 & $92 / 08$ \\
\hline 78 & PCT-11 $0 \backslash 012$, Bo $3 \backslash 1>1-M-3-1-1-1-\mathrm{M}$ & 8712 & $\mathrm{AB}$ & 16.7 & A-K & 19.5 & 56 & $92 / 08$ \\
\hline 86 & Fe de Arroz 50 & 8538 & $\mathrm{AB}$ & 16.5 & A-K & 21.1 & 58 & $90 / 10$ \\
\hline 41 & PCT-4|0|0|1>295-2-3-1-2-4 & 8439 & $\mathrm{AB}$ & 17.0 & $\mathrm{~A}-\mathrm{K}$ & 21.8 & 69 & $81 / 19$ \\
\hline 90 & Testigo LOCAL (INTA L-9) & 8434 & $A B$ & 16.3 & A-K & 20.8 & 66 & $90 / 10$ \\
\hline
\end{tabular}

*Medias con la misma letra son estadísticamente iguales, según TUKEY con $\mathrm{P}=0.05$.Rend: Rendimiento; ${ }^{\circ} \mathrm{H}$ : Humedad; \% CV: Porcentaje de Coeficiente de variación; R E/Q: Relación Entero Quebrado.

Segunda fase: Evaluación y selección en etapa de líneas avanzadas. En la segunda fase, evaluación y selección de líneas avanzadas de arroz, conformada por 25 líneas, se identificaron 12 materiales promisorios que superan las $8.8 \mathrm{t} \mathrm{ha-}^{1}$ (cuadro 2). El 16\% de los materiales introducidos fueron capaces de superar productivamente al testigo local de mejor rendimiento (INTA Dorado). Los materiales más productivos maduraron entre los 117 y 120 después de la emergencia (dde). Este grupo con madures intermedia, es un grupo estratégico desde el punto de vista de optimización del riego y de adaptación al cambio climático para condiciones de secano. Estos genotipos presentan las cualidades industriales exigidas por el comercio. 
Cuadro 2. Comportamiento agronómico e industrial de los mejores materiales productivos. Evaluación de líneas avanzadas de arroz, vivero TAINIC, período seco 2015

\begin{tabular}{|c|c|c|c|c|c|c|c|c|c|}
\hline \multirow{2}{*}{ Trat } & \multirow{2}{*}{ Genotipo } & \multicolumn{2}{|c|}{ Rend. $14 \%{ }^{\circ} \mathrm{H}$} & Madures & & \multicolumn{3}{|c|}{ Calidad del Arroz } & \multirow[b]{2}{*}{ Forma } \\
\hline & & & $\mathrm{kg} \mathrm{ha}^{1}$ & \multicolumn{2}{|c|}{ DDE } & $\%$ Oro & \%Ent. & R E/Q & \\
\hline 1 & CT19298-(100)-1-2-3-1-4MP & 10654 & $\mathrm{~A}^{*}$ & 120.3 & B-E* & 63 & 54 & $87 / 13$ & Alargado \\
\hline 6 & IR77430-14-B-1-2-B-3-4SR-1-M & 10246 & $\mathrm{AB}$ & 120.3 & B-E & 63 & 56 & $90 / 10$ & Alargado \\
\hline 13 & IR90154-53-2-1-M & 9698 & $\mathrm{ABC}$ & 115.5 & F-I & 73 & 71 & $98 / 02$ & Alargado \\
\hline 22 & IR 64 & 9639 & $\mathrm{ABC}$ & 114.3 & G-I & 63 & 60 & $96 / 04$ & Alargado \\
\hline 25 & INTA Dorado (TL) & 9291 & $\mathrm{ABC}$ & 125.0 & A & 59 & 52 & $89 / 11$ & Alargado \\
\hline 9 & IR75870-5-8-5-B-2-B-1SR-8-M & 9204 & $\mathrm{ABC}$ & 113.3 & H-J & 62 & 59 & $96 / 04$ & Alargado \\
\hline 8 & IR87233-19-4-2-2-M & 9154 & $\mathrm{ABC}$ & 110.3 & J & 65 & 61 & $94 / 06$ & Alargado \\
\hline 21 & Fe de Arroz 50 & 9178 & $\mathrm{ABC}$ & 122.3 & $\mathrm{ABC}$ & 64 & 59 & $94 / 06$ & Alargado \\
\hline 10 & IR87233-61-15-2-2-M & 8920 & $\mathrm{ABC}$ & 115.7 & F-I & 63 & 59 & $96 / 04$ & Alargado \\
\hline 7 & IR31917-45-3-2-1-1SR-5-M & 8912 & $\mathrm{ABC}$ & 117.5 & D-G & 59 & 48 & $83 / 17$ & Alargado \\
\hline 24 & INTA L-9 (TL) & 8835 & $\mathrm{ABC}$ & 119.7 & C-E & 66 & 61 & $94 / 06$ & Alargado \\
\hline
\end{tabular}

*Medias con la misma letra son estadísticamente iguales, según TUKEY con $\mathrm{P}=0.05$. Rend: Rendimiento; ${ }^{\circ} \mathrm{H}$ : Humedad; DDE: Días después de la emergencia; \% Oro: Porcentaje de arroz oro; \% Ent.: Porcentaje de arroz entero; R E/Q: Relación Entero Quebrado.
Los cuatro ambientes de secano (Pantasma, Jalapa, Posoltega y San Carlos), presentaron rendimientos medios inferiores a los obtenidos en condiciones de riego. En orden de importancia productiva se ubica en primer lugar el ambiente Jalapa, seguido de San Carlos, Posoltega y Pantasma, existiendo diferencias estadísticas entre sí. En Jalapa y San Carlos, las condiciones climáticas (precipitación) fueron óptimas para el cultivo; en cambio en los ambientes de Posoltega y Pantasma, las lluvias fueron deficitarias y erráticas. El rendimiento medio de los cuatro tratamientos productivos osciló entre 6.5 y 7.3 t ha- ${ }^{1}$.
El análisis de conglomerado con el criterio de aproximación del $25 \%$, permite agrupar a los genotipos en tres grupos con características en común (figura 3). El grupo de mayor importancia productiva lo conforman los genotipos: IR 64, IR87233-61-15-2-2-M, IR31917-45-3-2-1-1SR-5-M, IR77378-11-B-6-1-B-1SR-3-M, Fe de Arroz 50, IR9015453-2-1-M y CT19298-(100)-1-2-3-1-4MP. Este es un grupo notablemente superior al resto. Lo anterior se debe a que son de ciclo largo, son los más productivos y tienen mayor capacidad de macollamiento, granos por panícula y peso de grano. En un segundo grupo destacan los tratamientos 6, 25 y 9 , por ser altamente productivo y de ciclo intermedio, lo que sería importante para las condiciones de riego y secano.

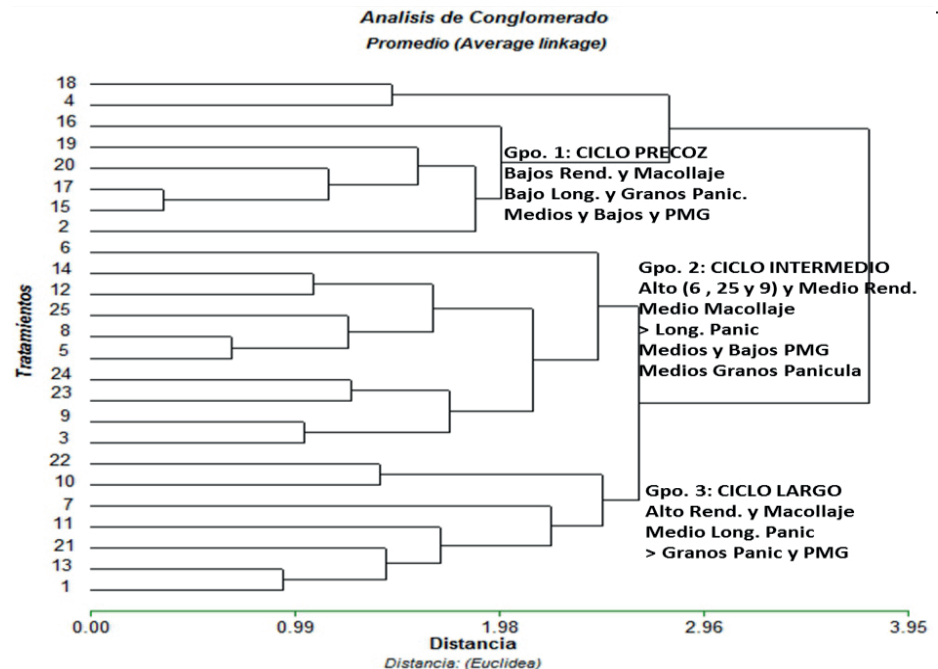

Figura 3. Análisis de conglomerado según componentes de rendimiento. TAINIC, período seco 2015.

Tercera fase: Evaluación multiambiental de rendimiento. Los ambientes de Darío y Masatepe fueron los más productivos y no existen diferencias estadísticas entre ellos (figuras 4). Ambos sitios superan significativamente a los rendimientos medios obtenidos por los otros cuatro ambientes (Pantasma, Jalapa, Posoltega y San Carlos). En Darío y Masatepe los genotipos fueron establecidos con riego y existen condiciones climáticas y de suelo optimas al cultivo.
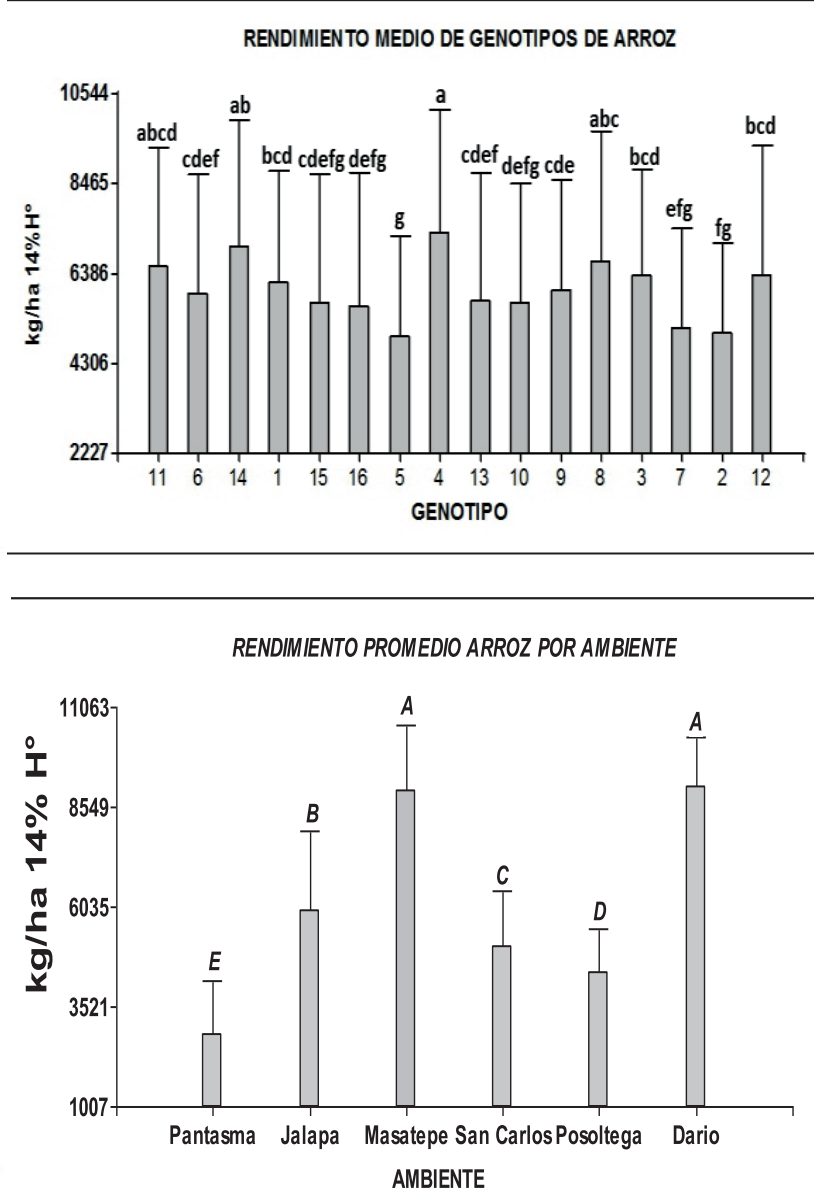

Figuras 4. Rendimientos medios por ambientes y genotipos, junio a noviembre del 2015.

El análisis de varianza indica diferencias altamente significativas por la interacción genotipo - ambiente. Los dos componentes principales explican el $72.1 \%$ de la variabilidad y por tanto se puede suponer que son suficientes para explicar los patrones debidos a la interacción.En los resultados del análisis de regresión por genotipo (GREG), los genotipos de comportamiento extremo (mejor o peor rendimiento) que 
definen el polígono envolvente (figura 5) están dados por los genotipos IR31917-45-3-2-1-1SR-5-M (4), CT18504-4-53Vi-2-3P (14), INTA L-9 (15) y IR87233-19-4-2-2-M (5).

(12) y IR77378-11-B-6-1-B-1SR-3-M (8). El cuadrante cuyo vértice está determinado por el genotipo IR87233-19-4-22-M (T5), involucra al sitio de Darío, en este se destacan los genotipos CT19298-(100)-1-2-3-1-4MP (1) y IR7743014-B-1-2-B-3-4SR-1-M (3). El cuadrante cuyo vértice está determinado el genotipo INTA L-9 (15), implica al sitio de Posoltega, destacando productivamente los genotipos IR75870-5-8-5-B-2-B-1SR-8-M (69 y CT16658-5-2-3SR-2-1-M (13).

\section{CONCLUSIONES}

Se seleccionaros cuatro genotipos (IR31917-45-3-2-1-1SR-5-M, CT185044-5-3Vi-2-3P, IR75870-5-8-5-B-2-B1SR-8-M y CT19298-(100)-1-2-3-14MP), que combinan características agronómicas, industriales y nutricionales deseables. Se estableció una relación positiva entre la cantidad de zinc disponible en el suelo y su influencia en la cantidad de zinc encontrada en grano pulido. Las variables que más influenciaron en la producción son en primer lugar días a madurez y longitud de panícula.

Se seleccionaron genotipos

Figura 5. Evaluación multi-ambiental de rendimiento, junio a noviembre del 2015.

Los genotipos IR31917-45-3-2-1-1SR-5-M (4) y CT18504-4-5-3Vi-2-3P (14), presentaron un mejor comportamiento productivo en Jalapa y Masatepe; y los genotipos IR87233-19-4-2-2-M (5) y INTÁ L-9 (15) resultados más bajos en Dario y Posoltega. En el cuadrante que tiene como vértice el genotipo IR31917-45-3-2-1-1SR-5-M (4), es un mega-ambiente que contiene a los sitios de Pantasma y Jalapa, comportándose los genotipos IR31917-45-3-2-1-1SR-5-M (4) y CT16344-CA-12-M (11) como los de mejor adaptación. El vértice que contiene al genotipo CT18504-4-5-3Vi-2-3P (T14) se comporta como un mega-ambiente conformado por los sitios de Masatepe y San Carlos, destacando los genotipos CT18504-4-5-3Vi-2-3P (T14), PCT-11/0/0/2,BO/1>66-1-7-1
(IR31917-45-3-2-1-1SR-5-M, CT18504-4-5-3Vi-2-3P, IR75870-5-8-5-B-2-B-1SR-8-M y CT19298-(100)-1-2-3-14MP), que presentan un comportamiento adaptativo amplio y específico para cada condición agroclimática y de manejo.

Se identificaron dos Mega-ambientes con genotipos en común. El primero formado por los sitios de Pantasma y Jalapa, destacándose el genotipo IR31917-45-3-2-1-1SR5-M (T4). El segundo mega-ambiente formado por los sitios de Masatepe y San Carlos destacándose el genotipo CT18504-4-5-3Vi-2-3P (T14). En los ambientes únicos de Posoltega y Darío los mejores genotipos fueron IR758705-8-5-B-2-B-1SR-8-M (T6) y CT19298-(100)-1-2-3-1-4MP (T1).

\section{REFERENCIAS BIBLIOGRÁFICAS}

Borrero, J. Sánchez, A. Cuasquer, J. Cabrera, J. Grenier, C. 2015. Evaluación del contenido de zinc en el grano pulido de líneas de arroz de riego y secano favorecido de Colombia. LX Reunión Anual PCCMCA en Ciudad Guatemala, Guatemala. CIAT- HP.

Goldschmidt, V.M. 1954. Geochemistry, Oxford Univ. Press, London, UK.

MAG. 2013. Dirección estadística del MAG. Managua, Nicaragua. Autor.

Martínez, C. 2007. Desarrollo de germoplasma con mayor valor nutricional para combatir la desnutrición en América Latina. LIII Congreso PCCMCA, Guatemala.

McClain, Craig J, Edward J, Kasarskis Jr and Allen JJ. 1985. Functional consequences of zinc deficiency. Progress in Food and Nutrition Science 9:185-226.

Nova, J. 2007. Hierro y zinc en el suelo y su expresión en el grano de arroz. Poster, CIAT- HARVESTPLUS-IDIAF, Republica Dominicana. Pachón, H. 2007. Conferencia Magistral sobre Biodisponibilidad de micros nutrientes en cultivos. LIII Congreso PCCMCA, Antigua-Guatemala.

Sandstead HH. 1985. Requirements of zinc in human subjects. Journal of the American College of Nutrition 4:73-82. 